\title{
NANOMATERIALS AND NANOTECHNOLOGY
}

November 18-19, 2021 I Webinar

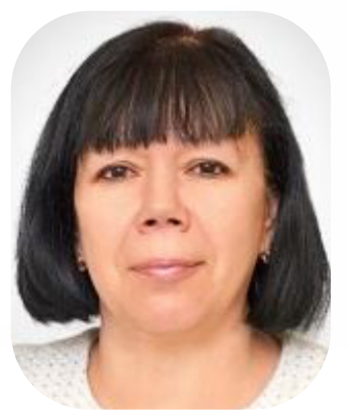

\section{Elena Liyaskina, Natalia Nazarova, Ekaterina Upyrkina, Alexandra Liyaskina, Victor Revin}

National Research Mordovia State University, Saransk, Russia

\section{Functional Biomaterials from Bacterial Nanocellulose}

$\mathrm{T}$ he current science of biomaterials aims at developing new materials with novel and multifunctional properties. Functional materials from bacterial nanocellulose (BNC) have received considerable research attention in recent times. BNC possesses unique structural, physical, chemical, mechanical, and biological features and has numerous applications in various fields, including biomedicine for tissue engineering, wound dressing, drug delivery, cosmetics, optoelectronics, bioprinting, biosensing, enzyme immobilization, environmental monitoring, etc. The subject of the experiment was nanocellulose, produced by bacterium Komagataeibacter sucrofermentans B-11267. The results show that the width of cellulose microfibrils formed by the K. sucrofermentans B-11267 strain averaged 40-90 nm. To reduce the cost of obtaining BNC, molasses and acidic by-products of the alcohol and dairy industries were used. A new BNCbased biocomposite material with adsorption properties in relation to fluoride ions was obtained. In addition, novel nanomaterials with antibacterial properties based on BNC and antibiotic sodium fusidate in the form of films, hydrogels and aerogels were obtained. The structure and physicochemical properties of nanomaterials were analyzed by SEM, FTIR spectroscopy and TGA. The antibacterial activities of nanomaterials were studied by the disc diffusion method. The results prove the potential of BNC-based biocomposites for biomedicine as wound dressing with high antibacterial activity and environmental monitoring as highly effective sorbents for fluoride. This work was supported by the Ministry of Science and Higher Education of the Russian Federation (grant number FZRS-2020-0003).

Keywords: biomaterials, bacterial nanocellulose, sorbents, wound dressing

\section{Biography:}

Dr. Elena Liyaskina, Associate professor, Department of Biotechnology and Biochemistry, Faculty of Biotechnology and Biology, National Research Mordovia State University, Saransk, Russia. Elena Liyaskina graduated as microbiologist in 1983, Kazan State University, Kazan, Russia. She received a PhD in biochemistry in 1995, Institute of Physiology and Biochemistry of Microorganisms, Pushchino, Moscow region, Russia. Elena Liyaskina is author of 5 books, 2 monographs, 10 patents and about 100 publications. Her research area of interests: microbiology, microbial biotechnology, bacterial exopolysaccharides, biopolymers, biocomposite materials. 